\title{
Enhanced Energy Efficient Multipath Routing Protocol for Wireless Sensor Communication Networks Using Cuckoo Search Algorithm
}

\author{
D. Antony Arul Raj' ${ }^{1}$ P. Sumathi ${ }^{2}$ \\ ${ }^{1}$ Department of Computer Science, VLB Janakiammal College of Arts and Science, Coimbatore, India \\ ${ }^{2}$ PG \& Research Department of Computer Science, Government Arts College, Coimbatore, India \\ Email: antonyarulraj.david@yahoo.com, sumathirajes@hotmail.com
}

Received 4 March 2014; revised 4 April 2014; accepted 11 April 2014

Copyright (C) 2014 by authors and Scientific Research Publishing Inc.

This work is licensed under the Creative Commons Attribution International License (CC BY).

http://creativecommons.org/licenses/by/4.0/

(c) (i) Open Access

\begin{abstract}
Energy efficient routing is one of the major thrust areas in Wireless Sensor Communication Networks (WSCNs) and it attracts most of the researchers by its valuable applications and various challenges. Wireless sensor networks contain several nodes in its terrain region. Reducing the energy consumption over the WSCN has its significance since the nodes are battery powered. Various research methodologies were proposed by researchers in this area. One of the bio-inspired computing paradigms named Cuckoo search algorithm is used in this research work for finding the energy efficient path and routing is performed. Several performance metrics are taken into account for determining the performance of the proposed routing protocol such as throughput, packet delivery ratio, energy consumption and delay. Simulation is performed using NS2 and the results shows that the proposed routing protocol is better in terms of average throughput, and average energy consumption.
\end{abstract}

\section{Keywords}

Wireless Sensor Communication Networks, Cuckoo Search Algorithm, AODV, AOMDV

\section{Introduction}

WSCNs are a fast growing and stimulating research area that has attracted significant research attention. WSCNs normally consist of hundreds or thousands of low powered sensor nodes that have ability to commu- 
nicate either directly to the Base Station (BS) or among each other [1] with capable of sensing, processing and transmitting environmental information (Figure 1). WSCNs are deployed to monitor certain physical phenomena or to detect and track certain objects in different domains including military, medical, environmental, industrial and home networks [2] [3]. Almost every sensor node consists of the following components [4].

1) Sensing devices;

2) Communication devices;

3) Controller;

4) Memory unit;

5) Energy supply unit.

In recent days science and engineering problem are solved by the computational algorithms inspired by biological processes. The evolutionary computation and swarm intelligence algorithms are derived based on the analogy of natural evolution and biological activities. The following biological activities are also included in the bio inspired techniques.

- Genetic algorithms;

- Genetic programming;

- Differential evolution;

- Particle swarm optimization;

- Ant colony optimization;

- Artificial neural networks, etc.

The bio inspired algorithms use the random-search techniques and heuristics to guide the search find the optimal solution and speed-up the convergence to obtain the global optimal solutions. While comparing the conventional optimization solver to the bio-inspired methods, the bio inspired methods have several attractive features and advantages. They also facilitate the advantage of simulation and optimization environment simultaneously to solve hard-to-define, real-world problems. These biologically inspired methods have provided novel ways of problem-solving for practical problems in traffic routing, networking, games, industry, robotics, economics, mechanical, chemical, electrical, civil, water resources and others fields [5].

Although many research works have been conducted on the performance and enhancement of the WSCNs, Bio inspired computing provides another dimension for enhancing the energy utilization of WSCNs. Bio inspired computing is used to find the optimal solution for the various problems in different areas of research. Recently the Bio inspired optimization techniques are adopted by different types of network researchers to enhance the life time of their network and proved that the biological species such as ant, bee, swarm as an analogy providing a natural model for combinatorial optimization problems [6]-[9].

\subsection{Problem Statement}

Even though all the Layer of the WSCNs uses the energy, consumption of energy in Network layer by the routing protocol is more. Hence constructing the Energy Efficient Routing Protocol becomes the primary goal of the recent day researches. Now days, Bio-inspired computing techniques are used to enhance the performance of the Energy Efficient routing protocols in an optimal way.

\subsection{Motivation and Objective}

The motive of this proposed research works is to increase the life time of the WSCNs, since the nodes in WSCNs are usually battery operated with limited energy resource and there is no option to change or replenish the batteries because nodes may deployed in a hostile or unpractical environment. Due to the low power batteries the utilization of the energy by the WSCNs, to be handle in an optimal manner, which increase the life time of the network. The main objective of this paper is to construct a new Enhanced Energy Efficient Multi path

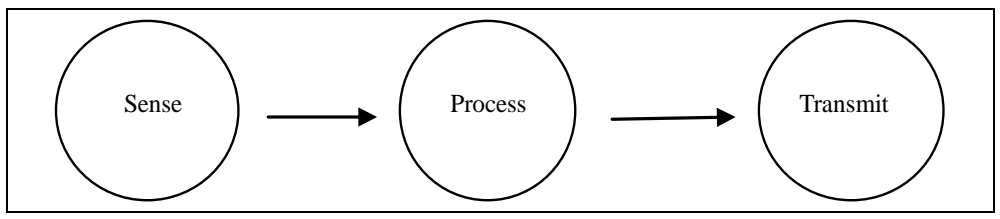

Figure 1. Activities of the sensor nodes. 
routing protocol using Bio-inspired Computing Techniques which used to produce better throughput, packet delivery ratio, and delay and energy consumption.

\subsection{Organization of the Paper}

This remainder of the paper is organized as follows: Section 2 reviews the background of the existing routing protocols. Section 3 describes the proposed work, about the methodologies followed and about the implementation details. Section 4 depicts the simulation settings and performance evolution of the proposed protocol. Section 5 shows results. Finally conclusions are given in the Section 6.

\section{Related Works}

Initially, Swarm intelligence is one of the bio-inspired computing was applied on graph theory as Artificial Intelligence approach to the Travelling Salesman problem using ants [10]. Then this technique becomes more popular and it was applied in communication network domain. It was used on packet routing initially with AntNet [11].

Ant Based Routing Algorithm for Manets (ARAMA) was used to send packets on Ad-hoc Networks the difference between this ARAMA and AntNet is that the amount of energy from nodes batteries influences the amount of pheromone laid on nodes within the path [12].

Ant-Based On Demand Energy Routing (AOER), was proposed to increase Ad-hoc networks' lifetime by adjust the routing technique. In AOER ants collect the remaining battery power of nodes, forward capability and hop counting information and this information are used to build an inverted probabilistic routing table (PRT). This PRT is used to find the less energy power path for future transmission [13].

Levi Bayde Ribeiro and Miguel Franklin were proposed Bio-inspired Optimization for Sensor Network Lifetime (Bio4Sel) based on Swarm Intelligence, more specifically on Ant Colonies. The proposed BioSel was compared with ARAMA and AODV [14].

Venkatesh Madhadevan and Frank Chiang proposed A Bio-inspired Power Efficient Routing Scheme for Sensor Networks (iACO) using a nature colonial scheme and proved that the delay, throughput and packet loss have been improved by iACO [15].

Energy balanced and based routing protocol (EBAB) proposed by Wang et al. They constructed an adaptive dynamic routing algorithm based on ant colony optimization. This EBAB was compared with LEACH and proved the better performance of EBAB [16].

Guo et al. [17] proposed Comprehensive routing protocol (CRP) based on Ant Colony algorithm, which is an improved version of (EAR) Energy Aware Routing. This protocol was designed in three phases: routing table, data communication and routing maintenance. CRP was implemented and compared with EAR and proved, its better performance with lack of QoS metrics.

Xiu-li et al. [18] proposed multipath routing based on ant colony systems for wireless sensor networks. It endues the ant with new characteristic and search method. The proposed protocol was compared with Ant colony system based routing (ACS) and proved that the average transmission delay was improved.

T. A. Ananth and N. Karthikeyan were proposed power-aware on-demand routing protocol called PAAOMDV, to increase lifetime of Sensor Networks. In the proposed protocol the source is capable to anticipate the traffic of the request, and each node on the route makes energy reservation based on the anticipated traffic. In view of the fact that a node knows not only its physical remaining energy, but also the expected energy possibly to be obsessive in the future, it will report its energy status more perfectly. A new cost utility is used in route selection, which takes both shortest-hop and maximum-lifetime into kindness. Results show that, the energy consumption in PA-AOMDV is more balanced than in the common on-demand protocols, and PA-AOMDV is with much longer system lifetime, along with higher delivery rate [19].

\section{Proposed Work}

In this section, a novel Enhanced energy efficient multi path routing protocol using cuckoo search algorithm, named EEEMRP, is presented. The EEEMRP extends the Ad-hoc On-Demand Multipath Distance Vector (AOMDV) routing protocol [20] by Cuckoo search algorithm.

\subsection{AOMDV}

AOMDV is, itself, an extension of the Ad-hoc On-Demand Distance Vector (AODV) routing protocol [21]. 
AOMDV uses distance vector concept and hop-by-hop routing approach. AOMDV also uses route discovery process to discover the on demand routes. The route request (RREQ) propagates multiple reserves between source and destination [19]. AOMDV also provides intermediate nodes with alternate paths as they are found to be useful in reducing route discovery frequency [22]. The main objective of the AOMDV protocol is to detect the multiple paths with loop-free and disjoints using flood-based route discovery. Entry expiration time gives the time after which, if a corresponding RREP has not been received, the entry is deleted. The routing table entry is modified for the maintenance of the multiple entries and multiple loop-free paths. Firstly, advertised hop-count replaces hop-count and advertised hop-count is the maximum over all paths from the current node to nd, so only one value is advertised from that node for a given destination sequence number. Secondly, next-hop IP address is replaced by a list of all next-hop nodes and corresponding hop-counts of the saved paths to nd from that node, as follows:

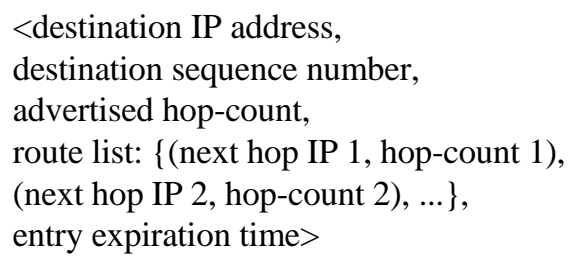

To obtain link-disjoint paths in AOMDV nd can reply to multiple copies of a given RREQ, as long as they arrive via different neighbors.

\subsection{Cuckoo Search Algorithm}

The Cuckoo search algorithm (CSA) is a meta-heuristic optimization algorithm to solve the problem and provides an optimal solution.

\subsection{Cuckoo Breeding Behavior}

The CSA was inspired by the breeding behavior of some cuckoo species for their re-production process. The cuckoo species lay their eggs in the nests of host birds, after evaluating the host bird's nest. The evaluation is carried over based on the colors and patterns of the eggs of a few chosen host species. This reduces the probability of the eggs being abandoned and, therefore, increases their re-productivity parasitic cuckoos often choose a nest where the host bird just laid its own eggs. The cuckoo eggs hatch slightly earlier than their host eggs. When the first cuckoo chick is hatched, his first instinct action is to evict the host eggs by blindly propelling the eggs out of the nest. This action results in increasing the cuckoo chick's share of food provided by its host bird [23]. The CSA models can be applied to various optimization problems. Cuckoo Search Algorithm is as follows:

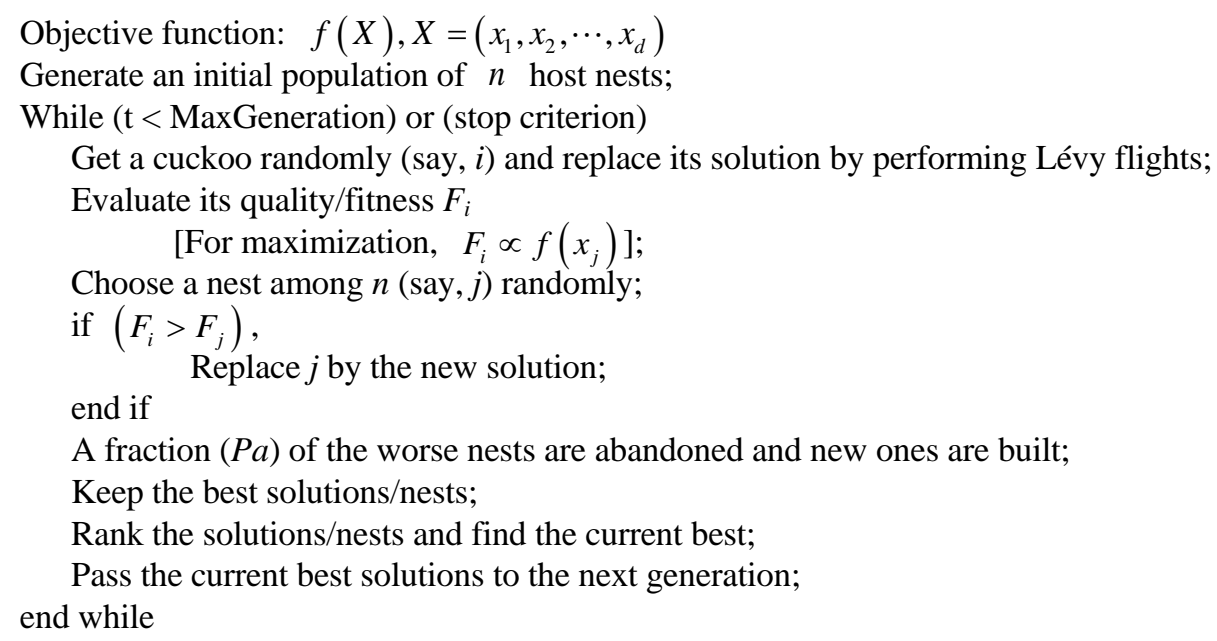




\section{Simulation Settings and Performance Metrics}

Ns-2 is used as a simulation platform and simulation setting is show in Table 1. The performance was evaluated based on Throughput and Energy consumption.

\section{Simulation Results}

Throughput is based on the number of packets and is compared between EEEMRP and AOMDV (EX). Results from Figure 2 shows that EEEMRP is better than AOMDV based on throughput. Figure 3 shows that EEEMRP has system lifetime than AOMDV even number of requests are increased.

\section{Conclusion}

In this paper, Cuckoo Search Algorithm is introduced in AOMDV so that optimal path along with maximum energy is established. Hence, based on the simulation results EEEMRP has better through put and system

Table 1. Simulation settings.

\section{Simulation Information}

\section{No of Nodes}

Area Size

Mac

$$
\text { Radio Range }
$$

Simulation Time

Traffic source

Packet Size

Mobility Model

Speed
50, 75, 100, 125 and 150

$1000 \times 1000$

802.11

$250 \mathrm{~m}$

50 sec

CBR

$512 \mathrm{~KB}$

Random Way point

$5 \mathrm{~m} / \mathrm{s}$

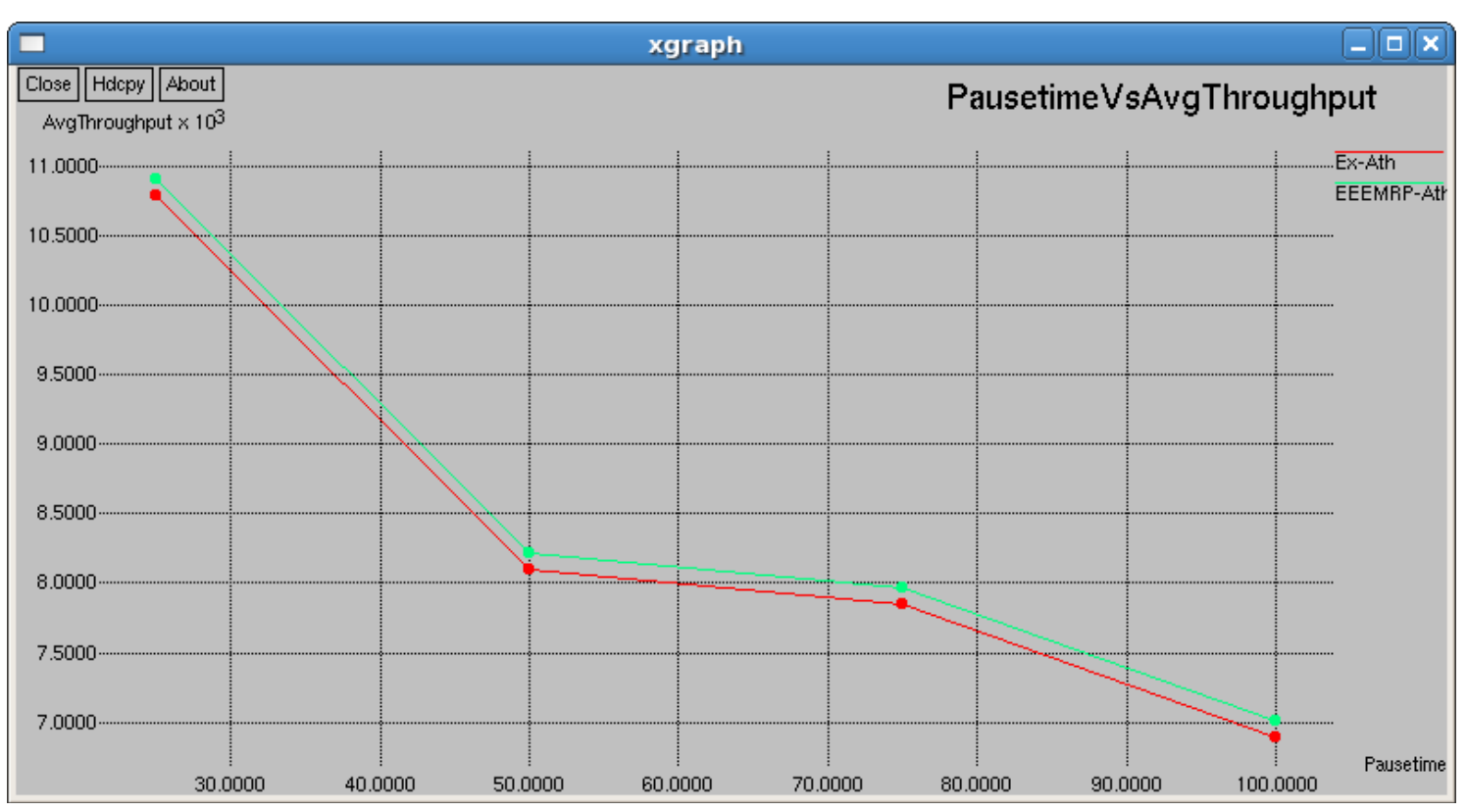

Figure 2. Comparison of throughput. 


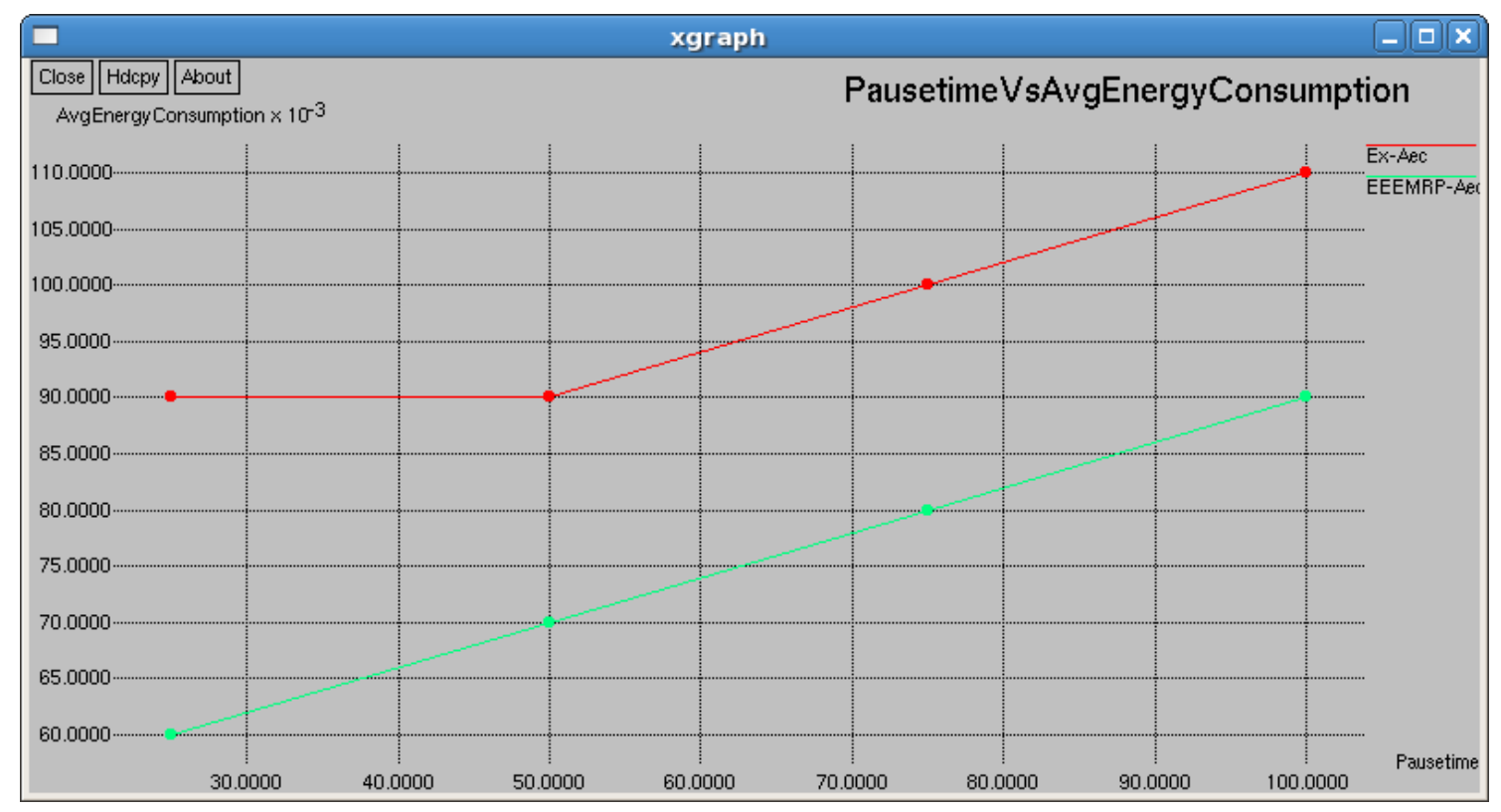

Figure 3. Comparison of energy consumption.

lifetime. EEEMRP controls routing with efficient energy. This energy efficiency can be established to other routing protocols in future.

\section{References}

[1] Abad, M.F.K. and Jamali, M.A.J. (2011) Modify Leach Algorithm for Wireless Sensor Networks. International Journal of Computer Science, 8, 219-224.

[2] Akyildiz, I.F., Su, W., Sankarasubramaniam, Y. and Cayirci, E. (2002) Wireless Sensor Networks: A Survey. Computer Networks, 38, 393-422. http://dx.doi.org/10.1016/S1389-1286(01)00302-4

[3] Chong, C.Y. and Kumar, S.P. (2003) Sensor Networks: Evolution, Opportunities, and Challenges. Proceedings of the IEEE, 91, 1247-1256. http://dx.doi.org/10.1109/JPROC.2003.814918

[4] Karl, H. and Willig, A. (2005) Protocols and Architecture for Wireless Sensor Networks. John Wiley \& Sons, Hoboken. http://dx.doi.org/10.1002/0470095121

[5] Janga Reddy, M. and Nagesh Kumar, D. (2012) Computational Algorithms Inspired by Biological Processes and Evolution. Current Science, 103, 370-380.

[6] Hussain, S. and Islam, O. (2008) Genetic Algorithm for Energy Efficient Trees in Wireless Sensor Networks. Springer, Boston, 1-14.

[7] Iyengar, S., Wu, H.C., Balakrishnan, N. and Chang, S.Y. (2007) Biologically Inspired Cooperative Routing for Wireless Mobile Sensor Networks. IEEE Systems Journal, 1, 29-37.

[8] Bonabeau, E. and Dorigo, M. (1999) Swarm Intelligence in Natural to Artificial Systems. Oxford University Press, London, 1-278.

[9] White, T. and Pagurek, B. (1998) Towards Mutli-Swarm Problem Solving in Networks. International Conference on Multi Agent System, Paris, 3-7 July 1998, 333-340.

[10] Dorgio, M., Maniezzo, V. and Colorni, A. (1991) The Ant System: An Autocatalytic Optimizing Process. Technical Report 91-016, Politecnico di Milano, Milano.

[11] Caro, G.D. and Dorigo, M. (1997) AntNet: A Mobile Agents Approach to Adaptive Routing. Technical Report IRIDIA/97-12, Universite Libre of de Bruxelles, Brussels.

[12] Hussein, O., Saadawi, T. and Lee, M.J. (2005) Probability Routing Algorithm for Mobile Ad Hoc Networks' Resources Management. IEEE Journal on Selected Areas in Communications, 23, 2248-2259. http://dx.doi.org/10.1109/JSAC.2005.857205

[13] Shuang, B., Li, Y., Li, Z. and Chen, J. (2007) An Ant-Based On-Demand Energy Routing Protocol for Ad Hoc Wireless Networks. WiCom'07: Proceedings of the International Conference on Wireless Communications, Networking and 
Mobile Computing, Shanghai, 21-25 September 2007, 1516-1519.

[14] Ribeiro, L.B. and de Castro, M.F. (2010) BioSel: A Bio-Inspired Routing Algorithm for Sensor Network Lifetime Optimization. International Conference on Telecommunications, 728-734.

[15] Mahadevan, V. and Chiang, F. (2010) iACO: A Bio-Inspired Power Efficient Routing Scheme for Sensor Networks. International Journal of Computer Theory and Engineering, 2, 972-977.

[16] Wang, G.F., Wang, Y. and Tao, X.L. (2009) An Ant Colony Clustering Routing Algorithm for Wireless Sensor Networks. Proceeding of 3rd International Conference on Genetic and Evolutionary Computing, Guilin, 14-17 October 2009, 670-673.

[17] Guo, W., Zhang, W. and Lu, G.A. (2010) A Comprehensive Routing Protocol in Wireless Sensor Network Based on Ant Colony Algorithm. Proceedings of the 2010 2nd International Conference on Networks Security Wireless Communications and Trusted Computing (NSWCTC), Wuhan, 24-25 April 2010, 41-44.

[18] Ren, X.L. Liang, H.W. and Wang, Y. (2008) Multipath Routing Based on Ant Colony System in Wireless Sensor Networks. Proceeding of International Conference on Computing Science and Sofrware Engineering, Wuhan, 12-14 December 2008, 202-205.

[19] Ananth, M.T.A. and Karthikeyan, N. (2012) On Demand Multipath Routing for Wireless Sensor Networks. International Journal of Advanced Information Science and Technology (IJAIST), 1, 86-91.

[20] Marina, M.K. and Das, S.R. (2001) On-Demand Multipath Distance Vector Routing in Ad Hoc Networks. Proceedings of 9th International Conference on Network Protocols, Riverside, 11-14 November 2001, 14-23.

[21] Perkins, C.E. and Royer, E.M. (1999) Ad-hoc On-Demand Distance Vector Routing. Proceedings of 2nd IEEE Workshop on Mobile Computing Systems and Applications, New Orleans, 25-26 February 1999, 90-100.

[22] Johnson, D.B. and Maltz, D.A. (1996) Dynamic Source Routing in Ad Hoc Wireless Networks. In: Imielinski, T. and Korth, H., Eds., Mobile Computing, Chapter 5, Kluwer Academic Publishers, 153-181. http://dx.doi.org/10.1007/978-0-585-29603-6_5

[23] Payne, R.B., Sorenson, M.D. and Klitz, K. (2005) The Cuckoos. Oxford University Press, Oxford. 